\title{
Collagenases and gelatinases in bone healing. The focus on mandibular fractures
}

\author{
Jacek Kurzepa ${ }^{1}$, Marcin Baran ${ }^{2}$, Slawomir Watroba ${ }^{3}$, Malgorzata Barud ${ }^{1}$, Daniel Babula ${ }^{4}$ \\ ${ }^{1}$ Department of Medical Chemistry, Medical University of Lublin, Poland \\ ${ }^{2}$ Department of Maxillofacial Surgery, Medical University of Lublin, Poland \\ ${ }^{3}$ Department of Obstetrics and Perinatology, Medical University of Lublin, Poland \\ ${ }^{4}$ Department of Neurosurgery and Neurotraumatology, Saint Edvige Provincial Hospital No 2 in Rzeszow, Poland
}

\begin{tabular}{|c|c|}
\hline ARTICLE INFO & ABSTRACT \\
\hline $\begin{array}{l}\text { Received } 17 \text { June } 2014 \\
\text { Accepted } 07 \text { August } 2014\end{array}$ & $\begin{array}{l}\text { Due to high amount of collagen fibres in the structure of bone, the enzymes capable } \\
\text { of collagen digestion play a key role in bone remodelling. Matrix metalloproteinases }\end{array}$ \\
\hline $\begin{array}{l}\text { Keywords: } \\
\text { MMP-1, } \\
\text { MMP-8, } \\
\text { MMP-13, } \\
\text { MMP-2, } \\
\text { MMP-9, } \\
\text { collagenases, } \\
\text { gelatinases, } \\
\text { mandibular fractures. }\end{array}$ & $\begin{array}{l}\text { proteins, e.g. collagen, proteoglycans, elastin or fibronectin. Among MMPs, collagenases } \\
\text { (MMP-1, MMP-8 and MMP-13) and gelatinases (MMP-2 and MMP-9) can cleave collagen } \\
\text { In addition, activity of the gelatinases (as an activation of proinflammatory cytokines) } \\
\text { facilitates spreading inflammation that is necessary during the first stage of bone healing. } \\
\text { Further studies related to the role of various MMPs in mandibular fractures should } \\
\text { precisely explain their function in the bone healing and evaluate the influence of MMPs } \\
\text { inhibitors on that process. This review provides the basic information about two groups } \\
\text { among MMPs family, collagenases and gelatinases, and their role in repairing processes } \\
\text { after mandibular fractures. }\end{array}$ \\
\hline
\end{tabular}

\section{INTRODUCTION}

The healing potential of bone, whether in a fracture or fusion model, is influenced by a variety of biochemical, biomechanical, cellular, hormonal, and pathological mechanisms.

A continuously occurring state of bone deposition, resorption, and remodeling, facilitates the healing process [17]. Matrix metalloproteinases (MMPs), prevailing extracellular endopeptideses, capable of digestion extracellularly located proteins, e.g. collagen, proteoglycans, elastin or fibronectin, are essential in bone healing process [46]. They play an important role in the physiological and pathological processes, as inflammation, cancer, cholesteatoma and other diseases e.g. osteoarthritis, multiple sclerosis, liver fibrosis, stroke and others $[21,28,46]$. Some of MMPs activate numerous biologically active compounds as chemokine CXCL-8, interleukin $1 \beta$ (IL-1 $\beta$ ), tumor necrosis

Corresponding author

e-mail: kurzepa@onet.pl factor $\alpha(\mathrm{TNF}-\alpha)$, interleukin $1 \beta$ ipso facto have an influence on several biological processes [21].

There is scant information in the literature about the relationship between MMPs activity and mandibular fractures. The phrase "matrix metalloproteinase mandibular fractures" contains just only 3 articles in PubMed database (November 2013), however, one of them is related to immature teeth disorder $[2,5,22]$. More publications are related to MMPs and bone fractures in general. This review provides the basic information about two groups among MMPs family, collagenases and gelatinases, and their role in repairing processes after mandibular fractures.

\section{CHARACTERISTICS OF MATRIX METALLO- PROTEINASES}

MMPs have been known since 1962, when two scientists found the first member of MMPs family in tadpole tails. The discovered enzyme was named collagenase [13]. The new enzyme, later called collagenase-1 (and finally MMP-1), is essential during the tadpole metamorphosis.

To this date, over twenty MMPs have been identified. The name of "metalloproteinases" comes from a zinc atom 
that is presented in enzyme active site. Zinc is necessary for catalysis and plays an active role in the peptide bound braking off. MMPs family is divided into several subgroups on the basis of their structure and specific substrates [11]:

1. collagenases (MMP-1, -8, -13),

2. gelatinases (MMP-2, -9),

3. stromelysins (MMP-3, -10, -11),

4. matrilysins (MMP-7, -26),

5. membrane-type matrix metalloproteinases (MT1, MT2, MT3, MT4, MT5, MT6-MMP)

6. others MMPs (MMP-12, -19, -20, -21, -23, -27, -28).

Due to their ability to the cleavage of peptide bond, the majority of MMPs are produced and secreted from cells in the inactive form (proenzymes). After activation their activity is regulated mainly by natural tissue inhibitors of MMPs (TIMPs) that can bind to the active side and block the substrate availability. To date, four types of tissue inhibitors of MMPs have been known and they are numbered from 1 to 4 [33].

A simplified structure of MMPs is presented in Figure 1. A signal peptide, propeptide and catalytic domain is the constant element of all MMPs. Membrane type MMPs have a transmembrane domain that anchors them to the cells surface. The hemopexin-like domain is similar to hemopexin - plasma protein responsible for heme transporting $[4,40]$. Collagenases and gelatinases have similar structure. Beside the constant elements, both types of enzymes contain hinge region and hemopexin-like domain [11]. In addition MMP-2 and MMP-9 have the hydrophobic fragment structurally resembling type II fibronectin that is involved in gelatine binding $[11,32,35,40]$. MMP-9 possesses a unique domain structurally similar to type V collagen [39].

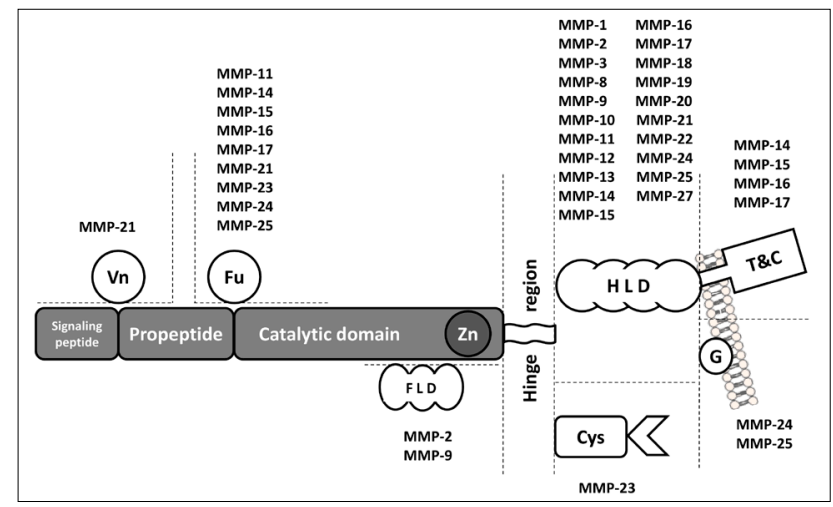

Figure 1. Simplified structure of MMPs. The gray colored domain are the constant constituent of all MMPs. $\mathrm{Zn}$ - zinc atom inside the active center, $\mathrm{Vn}$ - vitronectin region, $\mathrm{Fu}$ - furine like fragment, FLD - fibronectin like domain, HLD - hemopexin like domain, T\&C - transmembrane and cellular domains, G glycosylphosphatidyl inositol anchor, Cys - cysteine region with immunoglobulin like domain. Hinge region possess all MMPs with HLD or Cys.

\section{COLLAGENASES}

MMP-1 (collagenase-1), human fibroblast collagenase, has been the first vertebrate collagenase purified as a protein and cloned as a cDNA, and is considered the prototype for all the interstitial collagenases [37]. The MMP-1 has two molecular weights depending on presence or absence of carbohydrate residues; $57 \mathrm{kDa}$ (unglycosylated form) and 61 $\mathrm{kDa}$ (glycosylated). As other collagenases, MMP-1 cleaves type I, II and III collagens it has however the higher affinity to type III collagen. Additionally, such proteins as aggrecan, versican, perlecan, casein, nidogen, serpins, and tenascin- $\mathrm{C}$ are substrates for MMP-1 [30].

MMP-1 should be considered a multifunctional molecule because it participates also in the cleavage of a number of non-matrix substrates and cell surface molecules, that suggests a role in the regulation of cellular behavior [37]. MMP-1, as other MMPs, can proteolytically change the activity of various compounds via two ways; the cleavage of the blocking peptide (e.g. detaching of insulin-growth factor binding protein, activation of IL-1 $\beta$ ) or releasing the compound by digestion of extracellular matrix (e.g. FGF can be released after the perlecan digestion). MMP-1 plays an important role in such physiologic processes as development, tissue morphogenesis, and wound repair as well as it is involved in variety of human diseases including cancer, rheumatoid arthritis, pulmonary emphysema and fibrotic disorders [37].

MMP-8 (neutrophil collagenase-2) has $80 \mathrm{kDa}$ molecular weight as highly glycosylated proenzyme secreted from neutrophils. MMP-8 with low amount of carbohydrate residues is produced by fibroblasts, chondrocytes, endothelial cells, smooth muscle cells, and macrophages [24,50]. MMP-8 is more active against type I and III collagens. Besides them MMP-8 can digest type VII and X collagens, cartilage aggrecan, various proteoglycans, fibronectin, fibrinogen as well as non-structural substrates as: angiotensin, chemokines (MCP-1, MIG, IGFBP-5), L-selectin [50]. Previous studies suggest that MMP-8 is the predominant collagenase present in properly healing wounds, and that overexpression and activation of this collagenase may be involved in the pathogenesis of nonhealing chronic leg ulcers [1]. On the other hand the superphysiologic levels of the MMP-8 can result in decreased collagen and lead to impaired wound healing [7]. MMP-8 plays a role in the development of an inflammatory response, but it seems to exert an anti-inflammatory role during recovery and might be crucial for this process [8].

MMP-13 (collagenase 3) is $48 \mathrm{kDa}$ weight enzyme. Its expression is limited to situations in which rapid and effective remodeling of ECM proteins is necessary e.g. fetal bone development and adult bone healing. MMP-13 cleaves type II collagen more efficiently than types I and III. Other collagens digested by MMP-13 are type IX, X and XIV. Other proteins among ECM components that are MMP-13 substrates are: aggrecan, perlecan (simultaneously it can increase the biological activity of bFGF by detaching it from perlecan), large isoform of tenascin- $\mathrm{C}$ and fibronectin $[19,55]$. Finally, MMP-13 has an interaction with some clotting factors affecting clotting cascade. MMP-13 can digest also type IV collagen, the most significant substrate of both gelatinases. In addition MMP-13 has relatively high affinity to gelatin, a partially denatured collagen that is another important substrate of gelatinases [23]. 


\section{GELATINASES}

Among gelatinases, MMP-2 is a carbohydrate residuefree enzyme with molecular weight of $72 \mathrm{kDa}$ in pro-form. The pro-MMP-9 form has a molecular weight of $92 \mathrm{kDa}$, with the enzyme protein part constituting $76.2 \mathrm{kDa}$ [56].

The common substrates of gelatinases besides gelatine include: various native types of collagens, with type IV collagen forming the endothelial basement membrane, fibronectin, elastin, IL-1 $\beta$, transforming growth factor (TGF- $\beta$ ). MMP-9 is capable of activating chemokine CXCL-8 by proteolytic cleavage short polypeptide chain from CXCL-8 structure $[35,58]$. The effects of the activity of some biologically active compounds are associated with the capability of their proteolytic activation (e.g. IL-1 $\beta$ ) and increased bioavailability of these compounds due to the digestion of bonds with extracellular matrix proteins. Such a mechanism of activation has a vascular endothelial growth factor (VEGF) and TGF- $\beta$ that are activated by separation from the latency-associated protein (LAP). In addition, gelatinases are capable of proteolytic degrading myelin basic protein (MBP) [31].

Gelatinases, as other MMPs, are involved in the degradation of ECM components. However, their high affinity to collagen type IV, an important element of basement membranes, makes the gelatinases essential in the passage by inflammatory and tumor cells through endothelium, which leads to inflammation spreading.

The expression of MMP-9 occurs in numerous leukocytes, microglial cells, dendritic cells, fibroblasts, vascular endothelial cells, keratinocytes, and neoplastic cells $[27,35,41]$. The expression of MMP-2 takes place in microglial cells, adipocytes, astrocytes, vascular endothelial cells, macrophages, and smooth muscles [27,41].

\section{REGULATION OF COLLAGENASES GELATINASES ACTIVITIES}

AND

The activity of all MMPs is regulated at several levels: gene transcription, proenzyme activation, and through the action of natural inhibitors. Numerous biologically active compounds possess the ability to initiate collagenases and gelatinases expression. MMP-1 expression is trigged by epidermal growth factor (EGF), fibroblast growth factor (FGF) $-1,-2,-7,-9$, interferon- $\beta$ and $-\gamma$, IL- $1,-4,-5,-6$, chemokine CXCL-8, anti-inflammatory cytokine IL-10 and relaxin [18,37]. Vitamin A and retinoids decrease MMP-1 expression [54].

MMP-8 expression is up regulated by low doses of IL-1 $\beta$ and downregulated by TGF- $\beta 1[5,36]$.

Parathyroid hormone (PTH) is a key factor in calcium homeostasis and increases MMP-13 mRNA synthesis under in vivo and in vitro conditions [49,57]. Plateletderived growth factor (PDGF) and basic fibroblast growth factor (bFGF) have a similar activity to PDGF [23]. Both insulin-like growth factors (IGF I and II) inhibit MMP-13 expression. TGF- $\beta$ has ambiguous role related to MMP-13. It decreases the half-life of MMP-13 mRNA, however it increases MMP-13 expression. IL-1 $\beta$, TNF- $\alpha$, and IL-6 are the major cytokines that induce MMP-13 and MMP-9 expression $[23,49]$.

The additional activators of MMP-9 expression are adhesive molecules, lectins, some components of the extracellular matrix, extracellular matrix metalloproteinase inducer (EMMPRIN), and interactions among MMP9-producing cells [49]. The anti-inflammatory molecules, such as IL-4, IL-10, interferon $\beta$ (IFN- $\beta$ ), retinoids, and glucocorticosteroids can inhibit the MMP-9 expression [35].

MMPs, as other extracellular proteolytic enzymes, are secreted in the form of zymogens (proenzymes) to protect the cells against self-digestion. The thiol group of propeptide cysteine residue forms the coordinate bond with the zinc atom of the active centre what prevents binding of an appropriate substrate. The activation of gelatinases is the two-stage process. During the first stage, low-molecularweight compounds join to the sulphur within the propeptide. The examples of such compounds are mercury compound or nitric oxide, the endogenous activator of pro-MMP $[3,12,43]$. The inhibition of thiol group is reversible, the propeptide is not cleaved. This process is associated only with altered conformation of the enzyme, therefore, it was called the "cysteine switch" [41].

The next stage of activation is propeptide cleavage. This process is irreversible and is associated with a decrease of enzyme weight. MMP-13 plays the central role of numerous MMPs activation cascades. MMP-13 is activated by MT1-MMP, MMP-2 and MMP-3, as well as it catalyzes the activation of pro-MMP-2 and pro-MMP-9 [23].

Pro-MMP- 8 can be activated by variety of proteases, such as: cathepsin G, chymotrypsin and among MMPs members - MMP-3, -7, -10 and MT1-MMP (MMP-14) [50].

The activation of pro-MMP-9 under in vitro conditions occurs also in the presence of cathepsin G, chymotrypsin, trypsin, MMP-2, $-3,-10$, plasmin, and hyaluronic acid $[6,16,38]$. The in vivo activation of pro-MMP-2 is a complex process and it is mostly conducted on the surface of cells with the use of MT1-MMP (MMP-14) and TIMP-2 [42]. According to some other studies, the intergrin $\beta 1$ receptor is also likely to be involved in the activation of pro-MMP2 , the role of which in this case is similar to that of the MT1-MMP/TIMP-2 complex [44].

After the secretion from producing cells, MMPs activity can be regulated by natural tissue inhibitors of MMPs (TIMPs), the small proteins of 21-34 kDa molecular weights. TIMP-1 shows high affinity to MMP-1 and MMP-9, TIMP-2 to MMP-2, TIMP-3 to MMP-13 [3,23]. Besides inhibiting of enzymes, TIMPs significantly affect the induction of erythropoiesis (TIMP-1, -2), mitogenesis and apoptosis $[9,14,44]$.

Since MMPs contain a zinc atom in the catalytic domain and need calcium to functioning, a chelating compound may inhibit MMP activity. However, the usage of chelators as MMPs inhibitors is limited only to in vitro studies as highly unspecified therapy. Synthetic derivatives that mimic natural substrates were designed as MMP inhibitors. Such agents often belong to hydroxyamate - small molecule drug candidates characterized by an effective zinc-binding group plus additional side chains responsible for the selectivity [45]. Doxycycline, the antibiotic belonging to tetracycline group has also inhibitory potential against MMPs [25]. The 
inhibitors of 3-hydroxy-3-methylglutaryl coenzyme A reductase (statins) besides the lipid-lowering properties, decrease MMP-9 expression and biological activity [34].

\section{MMPS AND BONE FRACTURES.}

Bone tissue is a type of dense connective tissue with several functions as mechanical, synthetic and metabolic. Bone is composed of organic and inorganic elements. In $20 \%$ it is composed of water. The weight of dry bone is made up of inorganic calcium phosphate $(65-70 \%$ of the weight) and an organic matrix of fibrous protein and collagen (30-35\% of the weight). Osteoid is the unmineralized organic matrix secreted by osteoblasts. It is composed of $90 \%$ type I collagen and $10 \%$ ground substance, which consists of noncollagenous proteins, glycoproteins, proteoglycans, peptides, carbohydrates, and lipids. The mineralization of osteoid by inorganic mineral salts provides bone with its strength and rigidity [17].

Bone healing occurs in three stages: 1) the early inflammatory stage; 2) the repair stage; and 3) the late remodeling stage. In the first stage, a hematoma develops within the fracture site during the first few hours and days. Inflammatory cells, such as macrophages, monocytes, lymphocytes, polymorphonuclear cells and fibroblasts infiltrate the bone resulting in the formation of granulation tissue. The primary nutrient and oxygen supply of this early process is provided by the exposed cancellous bone and muscles. Early fracture healing is characterized by the initial formation of cartilage tissue in the callus, which is then resorbed by MMPs to allow for vascular invasion with the eventual replacement of cartilage with osseous tissue [53]. During the next stage fibroblasts begin to lay down a stroma that helps support vascular ingrowth. The last stage, remodeling, finishes the bone healing. The bone is restored to its original shape, structure, and mechanical strength. Remodeling of the bone occurs slowly over months to years and is facilitated by mechanical stress placed on bone [17].

MMPs, which can cleave native collagen at neutral $\mathrm{pH}$, play a critical role in the physiologic remodeling of bone. All collagenases digest type I, II and III collagens [11,20]. In addition gelatinases (MMP-2, and MMP-9) cleave partially denatured collagens (gelatin), native type IV collagen, that results totally degradation of collagen within the ECM.

Bone cells can produce various MMPs. MMP- 1 , MMP- 8 and MMP-9 are secreted by stromal fibroblasts, osteoblasts, and osteoclasts [20]. MMP-8 may be involved in articular cartilage turnover and cartilage pathophysiology associated with osteoarthritis. MMP-13 is specifically expressed in the cartilage of human osteoarthritis patients and is not present in normal adult cartilage [26]. However, MMP-13 together with MMP-9 is essential for normal skeletal development [48,51]. MMP-13 has been identified recently in matrix vesicles (MV) from growth plate cartilage, structures that have a key role in initiating the cartilage mineralization [23].

MMP-2 takes part in matrix turnover processes which is important for bone growth. In MMP-2-deficient adult mice the length of the tibia and femur is decreased, along with a reduced bone mineral density and trabecular bone quality [29]. In addition, MMP-2 participates in cartilage degradation. MMP-9 deficiency delays fracture healing with poor cartilage resorption and impaired capillary and chondroclast invasion. As mentioned above MMP-3 activates pro-MMP-9 during wound repair [22].

The exact role of MMPs in mandibular fractures is unclear. A few publications related to this issue are insufficient for forming the proper view. Therefore some pathological processes occurring during other bone healing have to be extrapolated to mandibular fractures healing. Similarly to other tissue injuries, bone fracture triggers an inflammatory response. Inflammation is a constant component of bone healing. In this matter inflammation has positive and negative role, however the molecular mechanism of inflammation effect on bone repair is still under investigation [10]. As mentioned above MMP-9 is key metalloproteinase essential for spreading of inflammatory process that could influence skeletal cell differentiation after fracture. The study performed by Wang et al. indicated that MMP-9 has indirect effects on skeletal cell differentiation by regulating the distribution of inflammatory cells, resulting in local regulation of periosteal cell differentiation [10,52].

For the examination of mandibular fractures an animal model was applied instead of commonly fractured in trauma because the last one undergoes multiple reconstructions in patients with congenital abnormalities, with a high variation in healing rates $(2 \%-32 \%)$ [22].

Lee et al. found that $\mathrm{CBP} / \mathrm{p} 300$-Interacting-Transactivator-with-ED-rich-tail-2 (CITED2) expression was inversely related to the expression of MMP-2, -3, -9, -13; over-expression of CITED2 in osteoblasts additionally inhibited the expression and activity of both gelatinases and MMP-3 and MMP-13 [22]. CITED2 is known to suppress genes mediating angiogenesis and ECM remodeling.

Bouletreau et al. investigated the role and mutual relationship between TGF- $\beta 3$ and TIMP- 1 in animal model of mandibular fractures [2]. They provided that TGF- $\beta 3$ expression was downregulated dramatically 3 days after the osteotomy and remained less than $20 \%$ of control levels throughout repair. TIMP-1 gene expression, low during early repair, is increased more than twofold over control at later time points.

\section{CONCLUSION}

Due to high amount of collagen fibres in the structure of bone the enzymes capable of collagen digestion play a key role in the bone remodelling. Collagenases and gelatinases together can cleave the collagen particle to forms that are able to undergo further intracellular break down. In addition activity of the gelatinases facilitates the spread of inflammation that is necessary during the first stage of bone healing. Further studies related to the role of various MMPs in mandibular fractures should precisely explain their function in the bone healing and evaluate the influence of MMPs inhibitors on that process.

\section{DECLARATION OF INTEREST}

The authors report no conflicts of interest. 


\section{REFERENCES}

1. Armstrong DG., Jude EB.: The role of matrix metalloproteinases in wound healing. J Am Pediatr Med Assoc., 92, 12, 2002.

2. Bouletreau P.J., Steinbrech D., Spector J.A. et al: Gene expression of transforming growth factor-beta 3 and tissue inhibitor of metalloproteinase type 1 during membranous bone healing in rats. J Craniofac Surg., 11, 521, 2000.

3. Brew K., Dinakarpandian D., Nagase H.: Tissue inhibitors of metalloproteinases: evolution, structure and function. Biochim Biophys Acta, 1477, 267, 2000.

4. Cha H., Kopetzki E., Huber R. et al. Structural basis of the adaptive molecular recognition by MMP9. J Mol Biol., 320, 1065, 2002.

5. Chubinskaya S., Huch K., Mikecz K. et al.: Chondrocyte matrix metalloproteinase-8: up-regulation of neutrophil collagenase by interleukin-1 beta in human cartilage from knee and ankle joints. Lab Invest., 74, 232, 1996.

6. Cuzner M.L., Gveric D., Strand C. et al.: The expression of tissue-type plasminogen activator, matrix metalloproteases and endogenous inhibitors in the central nervous system in multiple sclerosis: comparison of stages in lesion evolution. J Neuropathol Exp Neurol., 55, 1194, 1996.

7. Danielsen P.L., Holst A.V., Maltesen H.R. et al.: Matrix metalloproteinase- 8 overexpression prevents proper tissue repair. Surgery, 150, 897, 2011.

8. Dejonckheere E., Vandenbroucke R.E., Libert C.: Matrix metalloproteinase 8 has a central role in inflammatory disorders and cancer progression. Cytokine Growth Factor Rev., 22, 73, 2011.

9. Docherty A.J., Lyons A., Smith B.J. et al.: Sequence of human tissue inhibitor of metalloproteinases and its identity to erythroidpotentiating activity. Nature, 318, 66, 1985.

10. Duerr S., Stremme S., Soeder S., et al.: MMP-2/gelatinase A is a gene product of human adult articular chondrocytes and is increased in osteoarthritic cartilage. Clin Exp Rheumatol., 22, 603, 2004.

11. Fic P., Zakrocka I., Kurzepa J., Stepulak A.: Matrix metalloproteinases and atherosclerosis. Postepy Hig Med Dosw (Online), 65, 16, 2011.

12. Galazka G., Windsor L.J., Birkedal-Hansen H., Engler JA.: APMA (4-aminophenylmercuric acetate) activation of stromelysin-1 involves protein interactions in addition to those with cysteine-75 in the propeptide. Biochemistry, 35, 11221, 1996.

13. Gross J., Lapiere C.M.: Collagenolytic activity in amphibian tissues: a tissue culture assay. Proc Natl Acad Sci USA., 48, 1014, 1962.

14. Guedez L., Stetler-Stevenson W.G., Wolff L. et al.: In vitro suppression of programmed cell death of B cells by tissue inhibitor of metalloproteinases-1. J Clin Invest., 102, 2002, 1998.

15. Hatibović-Kofman S., Raimundo L., Zheng L. et al.: Fracture resistance and histological findings of immature teeth treated with mineral trioxide aggregate. Dent Traumatol., 24, 272, 2008.

16. Isnard N., Legeais J.M., Renard G., Robert L.: Effect of hyaluronan on MMP expression and activation. Cell Biol Int., 25, 735, 2001.

17. Kalfas I.H.: Principles of bone healing. Neurosurg Focus, 10, 7, 2001.

18. Kapila S., Xie Y., Wang W.: Induction of MMP-1 (collagenase-1) by relaxin in fibrocartilaginous cells requires both the AP-1 and PEA-3 promoter sites. Orthod Craniofac Res., 12, 178, 2009.

19. Knäuper V., Cowell S., Smith B. et al.: The role of the C-terminal domain of human collagenase-3 (MMP-13) in the activation of procollagenase-3, substrate specificity, and tissue inhibitor of metalloproteinase interaction. J Biol Chem., 272, 7608, 1997.

20. Krane S.M.: Is collagenase (matrix metalloproteinase-1) necessary for bone and other connective tissue remodeling? Clin Orthop Relat Res., 313, 47, 1995.

21. Kurzepa J., Bartosik-Psujek H., Suchozebrska-Jesionek D. et al.: Role of matrix metalloproteinases in the pathogenesis of multiple sclerosis. Neurol Neurochir Pol., 39, 63, 2005.

22. Lee J.Y., Taub P.J., Wang L. et al.: Identification of CITED2 as a negative regulator of fracture healing. Biochem Biophys Res Commun., 387, 641, 2009.

23. Leeman M.F., Curran S., Murray G.I.: The structure, regulation, and function of human matrix metalloproteinase-13. Crit Rev Biochem Mol Biol., 37, 149, 2002.
24. Lenglet S., Mach F., Montecucco F.: Role of matrix metalloproteinase-8 in atherosclerosis. Mediators Inflamm., 2013, 659282, 2013.

25. Li H., Ezra D.G., Burton M.J., Bailly M.: Doxycycline prevents matrix remodeling and contraction by trichiasis-derived conjunctival fibroblasts. Invest Ophthalmol Vis Sci., 54, 4675, 2013.

26. Li N.G., Shi Z.H., Tang Y.P. et al.: New hope for the treatment of osteoarthritis through selective inhibition of MMP-13. Curr Med Chem., 18, 977, 2011.

27. Loy M., Burggraf D., Martens K.H. et al.: A gelatin in situ-overlay technique localizes brain matrix metalloproteinase activity in experimental focal cerebral ischemia. J Neurosci Methods, 116, 125, 2002.

28. Madro A., Kurzepa J., Czechowska G. et al. Gelatinase activities and TIMP-2 serum level in alcohol cirrhosis and chronic pancreatitis. Curr Iss Pharm Med Sci., 26, 57, 2013.

29. Madsen D.H., Jürgensen H.J., Ingvarsen S. et al.: Differential Actions of the Endocytic Collagen Receptor uPARAP/Endo180 and the Collagenase MMP-2 in Bone Homeostasis. PLoS One, 8, e71261, 2013.

30. McCawley L.J., Matrisian L.M.: Matrix metalloproteinases: they're not just for matrix anymore! Curr Opin Cell Biol., 13, 534, 2001.

31. Mott J.D., Werb Z.: Regulation of matrix biology by matrix metalloproteinases. Curr Opin Cell Biol., 16, 558, 2004.

32. Murphy G., Knäuper V.: Relating matrix metalloproteinase structure to function: why the "hemopexin" domain? Matrix Biol., 15, 511, 1997.

33. Nagase H., Visse R., Murphy G.: Structure and function of matrix metalloproteinases and TIMPs. Cardiovasc Res., 69, 562, 2006.

34. Nomura S., Yoshimura K., Akiyama N. et al: HMG-CoA reductase inhibitors reduce matrix metalloproteinase- 9 activity in human varicose veins. Eur Surg Res., 37, 370, 2005.

35. Opdenakker G., Van den Steen P.E., Van Damme J.: Gelatinase B: a tuner and amplifier of immune functions. Trends Immunol., 22, 571, 2001.

36. Palosaari H., Wahlgren J., Larmas M. et al.: The expression of MMP-8 in human odontoblasts and dental pulp cells is down-regulatedby TGF-beta1. J Dent Res., 79, 77, 2000.

37. Pardo A., Selman M.: MMP-1: the elder of the family. Int J Biochem Cell Biol., 37, 283, 2005.

38. Peppin G.J., Weiss S.J.: Activation of the endogenous metalloproteinase, gelatinase, by triggered human neutrophils. Proc Natl Acad Sci USA., 83, 4322, 1986.

39. Polette M., Nawrocki-Raby B., Gilles C. et al. Tumour invasion and matrix metalloproteinases. Crit Rev Oncol Hematol., 49, 179, 2004.

40. Rowsell S., Hawtin P., Minshull C.A. et al.: Crystal structure of human MMP9 in complex with a reverse hydroxamate inhibitor. $J$ Mol Biol., 319, 173, 2002

41. Sang Q.X., Birkedal-Hansen H., Van Wart H.E.: Proteolytic and non-proteolytic activation of human neutrophil progelatinase $B$. Biochim Biophys Acta, 1251, 99, 1995.

42. Sato H., Takino T., Okada Y. et al. A matrix metalloproteinase expressed on the surface of invasive tumor cells. Nature, 370, 61, 1994.

43. Schmidt R., Bültmann A., Ungerer M. et al.: Extracellular matrix metalloproteinase inducer regulates matrix metalloproteinase activity in cardiovascular cells: implications in acute myocardial infarction. Circulation, 113, 834, 2006.

44. Seltzer J.L., Lee A.Y., Akers K.T. et al.: Activation of 72-kDa type IV collagenase/gelatinase by normal fibroblasts in collagen lattices is mediated by integrin receptors but is not related to lattice contraction. Exp Cell Res., 213, 365, 1994.

45. Serra P., Bruczko M., Zapico J.M. et al.: MMP-2 selectivity in hydroxamate-type inhibitors. Curr Med Chem., 19, 1036, 2012.

46. Smigielski J., Kołomecki K., Ziemniak P. et al.: Degradation of collagen by metalloproteinase 2 in patients with abdominal hernias. Eur Surg Res., 42, 118, 2009.

47. Stetler-Stevenson W.G., Bersch N., Golde D.W.: Tissue inhibitor of metalloproteinase-2 (TIMP-2) has erythroid-potentiating activity. FEBS Lett., 296, 231, 1992.

48. Stickens D., Behonick D.J., Ortega N. et al.: Altered endochondral bone development in matrix metalloproteinase-13 deficient mice. Development, 131, 5883, 2004. 
49. Uchida M., Shima M., Chikazu D. et al.: Transcriptional induction of matrix metalloproteinase-13 (collagenase-3) by $1 \alpha, 25$ dihydroxyvitamin D3 in mouse osteoblastic MC3T3-E1 cells. J Bone Min Res., 16, 221, 2001.

50. Van Lint P., Libert C.: Matrix metalloproteinase-8: cleavage can be decisive. Cytokine Growth Factor Rev., 17, 217, 2006.

51. Vu T.H., Shipley J.M., Bergers G. et al.: MMP-9/gelatinase B is a key regulator of growth plate angiogenesis and apoptosis of hypertrophic chondrocytes. Cell, 93, 411, 1998.

52. Wang X., Yu Y.Y., Lieu S. et al.: MMP9 regulates the cellular response to inflammation after skeletal injury. Bone, 52,111, 2013.

53. Weiss S., Zimmermann G., Pufe T. et al.: The systemic angiogenic response during bone healing. Arch Orthop Trauma Surg., 127, 989, 2009.

54. White L.A., Mitchell T.I., Brinckerhoff CE.: Transforming growth factor beta inhibitory element in the rabbit matrix metalloproteinase-1 (collagenase-1) gene functions as a repressor of constitutive transcription. Biochim Biophys Acta, 1490, 259, 2000.
55. Whitelock J.M., Murdoch A.D., Iozzo R.V., Underwood P.A.: The degradation of human endothelial cell-derived perlecan and release of bound basic fibroblast growth factor by stromelysin, collagenase, plasmin, and heparanases. J Biol Chem., 271, 10079, 1996.

56. Wilhelm S.M., Collier I.E., Marmer B.L. et al.: SV40-transformed human lung fibroblasts secrete a $92-\mathrm{kDa}$ type IV collagenase which is identical to that secreted by normal human macrophages. J Biol Chem., 264, 17213, 1989.

57. Witty J.P., Foster S.A., Stricklin G.P. et al.: Parathyroid hormoneinduced resorption in fetal rat limb bones is associated with production of the metalloproteinases collagenase and gelatinase B. J Bone Min Res., 11, 72, 1996.

58. Yu Y., Koike T., Kitajima S. et al.: Temporal and quantitative analysis of expression of metalloproteinases (MMPs) and their endogenous inhibitors in atherosclerotic lesions. Histol Histopathol., 23, 1503, 2008. 